\title{
Optimization Method of Process Parameters for Multi-axis NC Machining of Complex Surfaces
}

\author{
Mei Tian \\ College of Mechanical Engineering, Jilin Engineering Normal University \\ No. 3050 Kaixuan Road Kuancheng District, 130052 Changchun China \\ 32593829@qq.com
}

Keywords: Complex surface; Multi-axis; Numerical Control (NC) machining; Precision

\begin{abstract}
In the process of actual machining, there are many factors that can affect the precision of multi-axis machining of complex surface, such as process parameters, tool path, programming errors and so on. This paper proposes a new method-adaptive error compensation algorithms by combining the adaptive control method with the programming error control. This method, based on the theory of analytic geometry, is applied to modeling for controlling the precision of the multi-axis NC machining of complex surface, and combines some examples to obtain the ratio of processing time and the machining precision before and after the improvement.
\end{abstract}

\section{Introduction}

In today's society, the application of complex surface in the fields of aviation, automobile, shipbuilding, mold, toy and shoe making industry has become more and more widely.[1] So people's requirements of the machining precision is also getting higher and higher. In the traditional processing methods, different processes need to be processed on different equipment, multi axis machining can save this step, in the process of saving time and improve processing efficiency and processing accuracy.

\section{Influencing Factors of Multi Axis NC Machining Error of Complex Surface}

Multi-axis NC Milling Error Causes. CNC machining process is actually the process of interpolation, that is, the curve of the need to be processed into a lot of small parts, and then use the basic line to fit the curve or surface to be processed. Before processing, according to the geometric information and process information on parts of the drawings to prepare the corresponding procedures. Will process the input of NC machine tool, by the numerical control device control machine tool main movement speed, start, stop, the feed movement of the direction, velocity and displacement, as well as the tool to choose the exchange and the workpiece clamping and cooling and lubricating switches etc.[2] When machining surface due to the different surface accuracy and processing will it is discretized into a series of plane micro, because processing surface on the vector method is changing, so cutter axis vector is constantly changing, resulting in the track of cutter contact points is different from the first discrete curve segment, and produce error.

Error Accumulation in NC Machining Process. In complex surface machining process, the accuracy of generated influence factors and error sources there are many, such as process system accuracy of machine tools, machine tool motion accuracy, process system by vibration, heat deformation factors, CNC programming technology, types of tools, cutting tolerance and spacing, knife and retreat knife, knife, etc. And the error accumulation to a certain extent, it will seriously affect the surface quality of the parts to be processed, so, we should find ways to reduce the cumulative error, until the accuracy requirements are met. Error modeling and analysis is the main technical means to achieve this purpose.[3] 


\section{Adaptive Error Compensation Method and Its Algorithm}

Compensation Method. The key to realize the error compensation is the linear combination of the data parser to the interpolation data and the error data.[4] Numerical control device according to the input of the parts of the program information, between the starting point and end point curve segment is described by spatial data closely, thus forming the contour, for complex shape, if the direct generation algorithm will become very complex, and the amount of work a computer will be great.[5] The interpolation errors produced in the multi axis NC machining are nonlinear. There are many ways to reduce the error, such as the linear encryption method, the adaptive method of tool location and so on. In order to make the errors in the machining process within the allowable tolerance range, the knife site adaptive compensation method is adopted to make the cutter location close to the complex.

Compensation Algorithm. Error compensation is to create a new error to offset the original error of the current problem. [6]Taking the five axis $\mathrm{NC}$ milling machine of A and $\mathrm{B}$ as an example, the rotation axis is analyzed and calculated, and the method is applied to the following compensation algorithm.

In Fig. 1, the $\left(p_{w 0}, u_{w 0}\right)$ and $\left(p_{w 1}, u_{w 1}\right)$ are located adjacent to the cutter location data, corresponding to the $\left(p_{w 0}, u_{w 0}\right)$ of each linkage control axis motion position is $\left(X_{0}, Y_{0}, Z_{0}, A_{0}, B_{0}\right)$, corresponding to the $\left(p_{w 1}, u_{w 1}\right)$ movement position respectively is $\left(X_{1}, Y_{1}, Z_{1}, A_{1}, B_{1}\right)$, then each axis movement is:

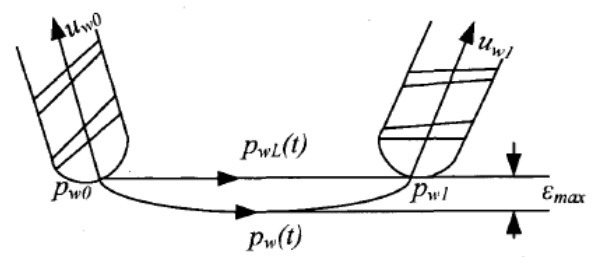

Fig. 1 Nonlinear error sample

$$
\left\{\begin{array}{l}
X(t)=X_{0}+t\left(X_{1}-X_{0}\right) \\
Y(t)=Y_{0}+t\left(Y_{1}-Y_{0}\right) \\
Z(t)=Z_{0}+t\left(Z_{1}-Z_{0}\right) \quad(0 \leq t \leq 1) \\
A(t)=A_{0}+t\left(A_{1}-A_{0}\right) \\
B(t)=B_{0}+t\left(B_{1}-B_{0}\right)
\end{array}\right.
$$

When the machine tool to do interpolation movement, the locus of the tool locus $p$ is $p_{w}(t)$ :

$$
\left[p_{w}(t), 1\right]^{T}=Q_{w, t}[X(t), Y(t), Z(t), A(t), B(t)] \cdot\left[p_{t}, 1\right]^{T}
$$

Programming linear $p_{w L}(t)$ direction vector

$$
a=\left(p_{w 1}-p_{w 0}\right) /\left(p_{w 1}-p_{w 0}\right)
$$

Then, the distance of any point on the $p_{w}(t)$ to $p_{w}(t)$ is $\varepsilon(t)$ :

$$
\varepsilon(t)=\left|p_{w}(t)-\left\{p_{w 0}+\left[\left(p_{w}(t)-p_{w 0}\right) \cdot a\right] \cdot a\right\}\right|
$$

$\varepsilon(t)$ of t derivative, obtained the maximum error $\varepsilon_{\max }$. If than allowed value, you need to insert a new cutter location in the knife two sites at the midpoint, and then in accordance with the method of calculation, error checking whether within the allowed range, if it is still not in the range, and then insert the new cutter location until it reaches accuracy requirements.[7] 


\section{Modeling of Multi Axis NC Machining Precision of Complex Surface}

Geometric Model of Spiral Cutting Edge of Ball end Mill.[8] The ball end milling cutter is developed on the basis of the end milling cutter, which is mainly used to process the surface of the mold cavity and the other forming surface.[9]

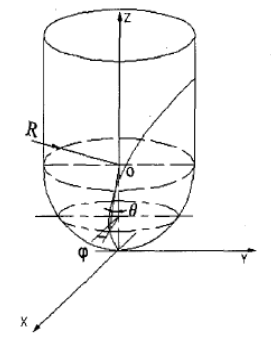

Fig. 2 Ball end milling cutter

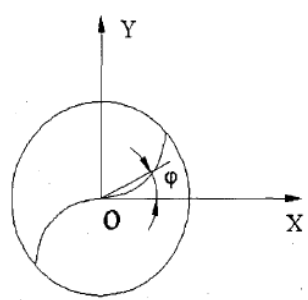

Fig. 3 2D cutter head

Coordinates of orthogonal helical surface equations:

$$
r=\left[\begin{array}{l}
x \\
y \\
z
\end{array}\right]=\left[\begin{array}{l}
R_{c} \cos \varphi \\
R_{c} \sin \varphi \\
P \varphi / 2 \pi
\end{array}\right]=\left[\begin{array}{c}
R \sin \theta \cos \varphi \\
R \sin \theta \sin \varphi \\
P \varphi / 2 \pi
\end{array}\right]
$$

Formula of coordinate system for spherical equation:

$$
r=\left[\begin{array}{c}
x \\
y \\
z
\end{array}\right]=\left[\begin{array}{c}
R_{c} \cos \varphi \\
R_{c} \sin \varphi \\
R(1-\cos \theta)
\end{array}\right]=\left[\begin{array}{l}
R \sin \theta \cos \varphi \\
R \sin \theta \sin \varphi \\
R(1-\cos \theta)
\end{array}\right]
$$

In the formula, $\mathrm{R}$ is the radius of the ball of the milling cutter; $\theta$ is cutting edge point and center line and cutter axis angle; $\varphi$ is the helix lag angle; $P$ is a spiral surface guide; $R_{c}$ is the distance between the cutting edge and the tool axis.

The equations (5) and (6) are obtained simultaneously.

$$
R(1-\cos \theta)=\frac{P \varphi}{2 \pi}
$$

Because $\tan \beta_{0}=\frac{2 \pi R}{P}$, then:

$$
\varphi=\tan \beta_{0}(1-\cos \theta)
$$

The edge line equation of the ball end mill is obtained

$$
r=\left[\begin{array}{l}
x \\
y \\
z
\end{array}\right]=\left[\begin{array}{c}
R \sin \theta \cos \left(\tan \beta_{0}(1-\cos \theta)\right) \\
R \sin \theta \sin \left(\tan \beta_{0}(1-\cos \theta)\right) \\
R(1-\cos \theta)
\end{array}\right]
$$

\section{Application Examples}

In order to compare the changes in the size of the workpiece machining error before and after adaptive compensation, do two sets of experiments. Section of the design surface is a sine curve, tool path planning, determine the cutting parameters. In the first set of experiments using CAXA 
Manufacturing Engineer in the automatic generation of $\mathrm{NC}$ code and in the second set of experiments using error compensation to rewrite the $\mathrm{NC}$ code, taking the mouse as an example to get the precision ratio of the optimization.

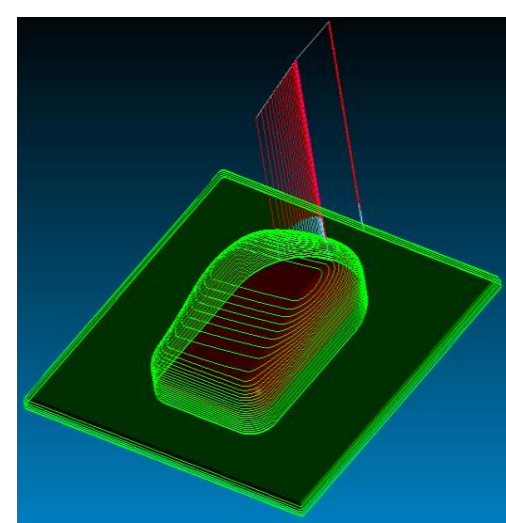

Figure5.Tool path diagram

Tabel 1 Accuracy comparison of parts

\section{Deviation $(\mathrm{mm}) \quad$ Before improvement After improvement}

Maximum deviation $\quad+0.162 /-1.040 \quad+0.144 /-0.087$

Minimum deviation $\quad+0.053 /-0.045 \quad+0.050 /-0.029$

standard deviation
0.100

0.052

In this paper, the influence factors of complex surface NC machining errors are analyzed, including the influence of programming errors on machining accuracy. In addition, the characteristic of NC machining process system and the precision of the numerical control system are also important factors that affect the machining accuracy. [10]The data in the table show that the algorithm proposed in this paper is feasible and effective.

\section{References}

[1] Y.J. Chen and X.T. Chen: Modular Machine Tool \& Automatic Manufacturing Technique,(2013) No.3,p96. (In Chinese)

[2] J.K. Yang: Management and technology of small and medium sized enterprises,(2014)No.06,p.248.(In Chinese)

[3] Q. Huang and C.J. Gao: China Mechanical Engineering, Vol. 25 (2014) No.7, p.857.

[4] K. Ren, W.H. Chen, J. Pan, H.G. Chen and S.D. Lin: Journal of Mechanical Engineering, Vol. 46 (2010) No.15, p.155. (In Chinese)

[5] C.H. Yu: Occupation, (2013) No.26, p.248.(In Chinese)

[6] J.G. Yang, Y.Q. Ren, W.B. Zhu, M.L. Huang and Z.H. Pan: Chinese Journal of Mechanical Engineering, Vol. 39 (2003) No.3, p.81. (In Chinese)

[7] .F. Ji, L.S. Zhou, L.L. An and C. Zhang: Journal of Chongqing University, Vol.33, p.37. (In Chinese)

[8] B.Yan,A.P.Xu,D.W.Zhang and T.Huang: Chinese Journal of Mechanical Engineering, (2002) No.02, p.160.(In Chinese)

[9] M.Q. Sun and Z.Y. Weng: Tool technology, vol.40 (2006) No.9, p7. (In Chinese)

[10]R.Q. He, S.J. Yan and Y.F. Zhou: Machine Tool \& Hydraulics, (2006) No.9, p80. (In Chinese) 